\title{
Composite coating with synergistic effect of biomimetic epoxy thermoset morphology and incorporated superhydrophobic silica for corrosion protection
}

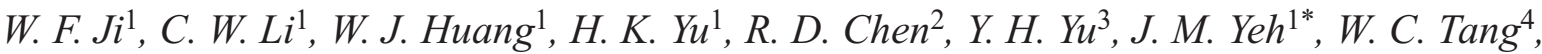 \\ Y. C. Su \\ ${ }^{1}$ Department of Chemistry, Center for Nanotechnology and Center for Biomedical Technology at Chung-Yuan Christian \\ University (CYCU), 32023 Chung Li, Taiwan, Republic of China \\ ${ }^{2}$ Master Program in Nanotechnology and Center for Nanotechnology at CYCU, 32023 Chung Li, Taiwan, Republic of \\ China \\ ${ }^{3}$ Department of Chemistry, Fu Jen Catholic University, Hsinchung Dist., 24205 New Taipei City, Taiwan, Republic of \\ China \\ ${ }^{4}$ Division of Applied Chemistry, Material and Chemical Research Laboratories, Industrial Technology Research Institute, \\ 30011 Hsinchu, Taiwan, Republic of China
}

Received 18 April 2016; accepted in revised form 27 June 2016

\begin{abstract}
In this work, potential anticorrosive coating resulted from the composite with synergistic effect of biomimetic epoxy thermoset (BET) morphology and incorporated superhydrophobic silica microspheres was presented. First of all, superhydrophobic methyl-modified silica (MS) microspheres were synthesized by performing the conventional base-catalyzed sol-gel process of MTMS and APTMS. The as-prepared MS microspheres were identified as having an average particle size of $\sim 1 \mu \mathrm{m}$ in diameter. The as-prepared MS microspheres were characterized by Fourier transform infrared spectrometry (FTIR), ${ }^{29} \mathrm{Si}$ and ${ }^{13} \mathrm{C}$ solid-state nuclear magnetic resonance (NMR) spectroscopy. Morphological properties of MS microspheres and BET-silica composite coating were studied by scanning electron microscopy (SEM). Subsequently, 3 wt $\%$ of MS microspheres were incorporated into an epoxy slurry of DGEBA/T-403 in dimetyl acetamide (DMAc), followed by performing the programmed heating through nanocasting technique with PDMS as soft template materials for pattern transfer by using leaf of Xanthosoma Sagittifolium as natural template, leading to the formation of artificial biomimetic composite coating. The appearance/dispersion capability of silica microspheres in BET coating was confirmed by the energy dispersive X-ray spectroscopy (EDX) and Si-mapping. The roughness level of BET and BEC-3\% were detected by AFM. The BETsilica composite was found to exhibit a contact angle (CA) of $\sim 153^{\circ}$, revealing the synergistic effect of biomimetic epoxy morphology and incorporated superhydrophobic MS microspheres, which is found to be more hydrophobic than that of neat epoxy thermoset $\left(\mathrm{CA}=81^{\circ}\right)$. Corrosion protection of as-prepared coatings was demonstrated by performing a series of electrochemical measurements (Tafel, Nyquists and Bode plots) upon CRS electrodes in saline condition. It should be noted that the BET coatings upon CRS electrode revealed an effectively enhanced corrosion protection as compared to that coatings without biomimetic morphology. Moreover, the BET coating with superhydrophobic MS microspheres upon CRS electrode was found to exhibit better corrosion protection as compared to a counterpart coating without MS microspheres.
\end{abstract}

Keywords: polymer composites, biomimetic, methyl-modified silica microsphere, epoxy resin, corrosion protection

\section{Introduction}

Corrosion is a significant research area for finishing of metallic industry. Therefore, protective organic/ polymeric coatings are typically utilized as a primer to protect metal surface from corrosion [1-3]. In the past decades, different classic polymers, such as

$\overline{{ }^{*} \text { Corresponding author, e-mail: juiming@,cycu.edu.tw }}$

C BME-PT 
epoxy resin, $[4,5]$ polyurethane $[6,7]$ and polyimide $[8,9]$ have been used by the environmentally sheltered coatings to enhance the anticorrosive effect upon various metallic substrates. In order to facilitate the corrosion protection of various metallic substrates, versatile alternative practical coatings are being continuously developed such as conducting/ electroactive polymers, $[10,11]$ conventional polymers containing filler with large aspect ratio $[12,13]$ and polymer coatings with superhydrophobic surface $[14,15]$.

Recently, great attention is paid to the potential of applications on bio-mimetic materials with super-hydrophobic surfaces structure due to their diverse potential and unprecedented hierarchical surfaces [1626]. Moreover, the studies of anticorrosive polymer coatings with roughly superhydrophobic or biomimetic surface attracted intensive research interests [2734]. For example, Liu et al. [35] found that the surface of copper block was found to display superhydrophobicity after treatment of n-tetradecanoic acid solution for a period of time, leading to the as-formed surface with better anticorrosion performance. Moreover, Wang et al. [36] demonstrated that the steel surface treated with specific surface modification to give coating with micro-/nano- hierarchical superhydrophobic structures upon steel, leading to an excellent corrosion protection property. Furthermore, Yang et al. [37] reported that the eletroactive polymer with the biomimetic topography also showing superhydrophobicity, which exhibited superior anticorrosive ability based on a series of electrochemical investigations in $3.5 \mathrm{wt} \% \mathrm{NaCl}$ solution. Enhancement of corrosion protection of above-mentioned superhydrophobic coatings may be attributed to the trapped gas within the valleys between the hills of the superhydrophobic surface [35].

On the other hand, the research activity in terms of polymer coating incorporated with hydrophobic inorganic particles has also evoked great research interests [38-43]. For example, Sun et al. [44] had explored that the superhydrophobic surfaces covered with cauliflower-like cluster binary micro-nano coatings and promoted excellent corrosion resistance property in the $3.5 \mathrm{wt} \% \mathrm{NaCl}$ solution. Moreover, Weng et al. [45] demonstrated that the as-prepared organic-inorganic superhydrophobic composite coating was found to boost the corrosion resistance property in saline condition (e.g., $3.5 \mathrm{wt} \% \mathrm{NaCl}$ solution) based on a series of electrochemical corrosion measurements. Chang et al. [46] found that the UV-cured biomimetic composites containing thermoplastic PMMA and micro-silica particles was found to give the coating surface with increased roughness, which may effectively increase the superhydrophobicity and therefore facilitate the corrosion resistance of metallic substrates in saline.

In this work, the synergistic effect of biomimetic morphology and incorporated superhydrophobic silica microspheres is used to prepare the BET-silica composite anticorrosion coatings. First of all superhydrophobic methyl-modified silica (MS) microspheres were synthesized by sol-gel reactions and characterized by FTIR, ${ }^{13} \mathrm{C}$ and ${ }^{29} \mathrm{Si}$ NMR spectra. Moreover, nanocasting technique was utilized with PDMS (poly dimethyl siloxane) as pattern-transfer polymer by using natural leaf of Xanthosoma Sagittifolium as natural template, leading to the formation of artificial BETsilica composite coatings. Furthermore, $3 \mathrm{wt} \%$ of asprepared superhydrophobic MS microspheres was added into BET to give the BET-silica composite coatings. SEM and contact angle measurements were applied to investigate the morphology and hydrophobicity of coating surface. Finally, anticorrosion performance of the developed BET-silica composite coatings upon CRS electrodes was evaluated by a series of electrochemical measurements (Tafel Nyquists and Bode plots) in $3.5 \mathrm{wt} \% \mathrm{NaCl}$ aqueous solution.

\section{Experiment}

\subsection{Materials and measurements}

In this study, the bisphenol A diglycidyl ether (DGEBA; Aldrich; Japan) and trimethylolpropanetris [poly(propylene glycol), amine terminated] ether ( $\mathrm{T}$ 403; Aldrich; American) were used as received without further treatment. Trimethoxy (methyl) silane (MTMS; Aldrich, 98.0\%; American), (3-aminopropyl) trimethoxysilane (APTMS; Fluka, 98.0\%; Switzerland) were used as sol-gel precursor and epoxy coupling agent, respectively. Ethanol (EtOH; Aldrich, 95\%; American), ammonium hydroxide solution $\left(\mathrm{NH}_{4} \mathrm{OH}\right.$; Aldrich, 28.0-30.0\%; American), polyvinylpyrrolidone (PVP, $M_{\mathrm{w}}=40,000$, polymeric stabilizer, Aldrich), and $N, N$-dimethyl acetamide (DMAc; 99.0\%, Aldrich; American) were used as received without further purification. The liquid com- 
ponents (Sylgard 184) of polydimethylsiloxane (PDMS; American) were supplied by Dow Corning Corporation. All reagents were reagent grade unless otherwise stated.

Fourier-transform infrared (FTIR) spectra were recorded using an FTIR spectrometer (JASCO FT/IR4200) operating at room temperature. The $X$-ray photoelectron spectroscopy XPS measurement was performed on Microlab MKII electron spectrometer with a base pressure of $10^{-10}$ Torr. The Sorvall RC-5C Plus Super-speed Centrifuge was used to separate methyl-modified silica (MS) particles by centrifugal force (7500 rpm). Both ${ }^{13} \mathrm{C}$ and ${ }^{29} \mathrm{Si}$ MAS solid-state NMR experiments were performed on a $400 \mathrm{MHz}$ solid-state NMR spectrometer. ${ }^{13} \mathrm{C}$ MAS NMR spectra were obtained at $100.63 \mathrm{MHz}$ with $7 \mathrm{kHz}$ applying $90^{\circ}$ pulses and $2.0 \mathrm{~s}$ pulse delays. To enhance carbon sensitivity, cross-polarization (CP) techniques were employed. ${ }^{29} \mathrm{Si}$ MAS NMR spectra were recorded at $79.49 \mathrm{MHz}$ applying $90^{\circ}$ pulses, $300 \mathrm{~s}$ pulse delays, and $5.0 \mathrm{~ms}$ contact time, with samples in $5.0 \mathrm{~mm}$ zirconia rotors spinning at $7 \mathrm{kHz}$. Surface morphologies of the superhydrophobic samples were observed by using SEM (JOEL JSM-7600F). Silica dispersion was detected by EDX (OxFord xmax 80). Contact angles were measured using a First Ten Angstroms FTA 125 at ambient temperature. Water droplets (about $4 \mu \mathrm{L}$ ) were carefully dropped onto the surfaces of samples, and contact angle was determined from the average of five measurements at various positions on the samples surface. Corrosion potential and corrosion current of sample-coated CRS electrodes were electrochemically measured using a VoltaLab 50 potentiostat/galvanostat. Electrochemical impedance spectroscopy (EIS) study was recorded on AutoLab (PGSTAT302N) potentiostat/galvanostat electrochemical analyzer.

\subsection{Synthesis of methyl-modified silica (MS) microsphere}

The MS microspheres were synthesized via conventional base-catalyzed sol-gel reactions of MTMS in the presence of APTMS molecules. A typical procedure to prepare the MS microspheres was given as follows: the surfactant (PVP, $0.1 \mathrm{~g}$ ) and $\mathrm{NH}_{4} \mathrm{OH}$ $(0.1 \mathrm{~mL})$ was dissolved in $40 \mathrm{~mL}$ of water and kept at $5^{\circ} \mathrm{C}$. The mixture of $2.04 \mathrm{~g}$ MTMS (20 mmole) and $0.136 \mathrm{~g}$ APTMS ( $1 \mathrm{mmole}$ ) was added drop-wise to the aqueous solution for about 1 hour at $5^{\circ} \mathrm{C}$. The solgel reaction was continued under magnetic stirring for 6 hours at room temperature. Finally, the as-prepared silica microspheres were washed repeatedly with ethanol $(\mathrm{EtOH})$ and separated from the aqueous mixture by centrifugal sedimentation of $7500 \mathrm{rpm}$ to remove the surfactant and re-dispersed in water.

\subsection{Preparation of PDMS template}

The PDMS pre-polymer was obtained by mixing the elastomer base and a curing agent at a specific weight ratio $(10: 1, \mathrm{w} / \mathrm{w})$. The PDMS pre-polymer was poured into a mold of $6 \times 6 \mathrm{~cm}^{2}$ fixed to a piece of fresh, natural Xanthosoma sagittifolium leaf (veins of leaf were removed in area of about $6 \times 6 \mathrm{~cm}^{2}$ ) and cured in oven programmed at $60^{\circ} \mathrm{C}$ for 6 hours. After curing process, the PDMS blocks were detached from the molds, to be used as a negative template for the following nanocasting process of pattern transferring [47].

\subsection{Preparation of CRS electrode coated with biomimetic epoxy thermoset (BET)}

The $1.024 \mathrm{~g}$ of DGEBA was dissolved in $5 \mathrm{~g}$ of DMAc under stirring for 1 hour, followed by adding $0.88 \mathrm{~g}$ of T-403 functioning as curing agent under stirring for 12 hours. Finally, the solution was cast onto the PDMS template, and then the cold-rolled steel (CRS) electrode was pressed against upon the surface of as-prepared epoxy slurry. Subsequently, the thermal curing process of epoxy thermoset was programmed at $50^{\circ} \mathrm{C}$ for 1 hour, $120^{\circ} \mathrm{C}$ for 2 hours and $140^{\circ} \mathrm{C}$ for 0.5 hour. The CRS electrode coated with BET can be obtained by detaching from PDMS template.

\subsection{Preparation of CRS electrode coated with biomimetic epoxy-silica composite (BEC-3 wt\%)}

The representative procedure for the preparation of CRS electrode coated with biomimetic epoxy-silica composite (BEC) was given as follows: $1.024 \mathrm{~g}$ of DGEBA and $0.075 \mathrm{~g}$ of the as-prepared MS particles were dissolved or dispersed into $5 \mathrm{~g}$ of DMAc under magnetic stirring for 1 hour, followed by introducing $0.5 \mathrm{~g}$ of T-403 for 6 hours under stirring. The mixed solution was eventually dropped and cast onto the PDMS template, the CRS electrode was then pressed 
against the surface of mixing slurry. Subsequently, the thermal curing process of $\mathrm{BEC}$ was programmed at $50^{\circ} \mathrm{C}$ for 1 hour, $120^{\circ} \mathrm{C}$ for 2 hours and $140^{\circ} \mathrm{C}$ for 0.5 hour. The CRS electrodes coated with BEC was then obtained by detaching from the PDMS template.

\subsection{Electrochemical corrosion studies}

Epoxy thermoset coatings of about $30 \mu \mathrm{m}$ in thickness were obtained after drying in a fume hood for 24 hours. Edges of the coupons were locked on the artificial electrode for corrosion testing. The coated and uncoated coupons were connected to the working electrode of an electrochemical cell. For the anticorrosion measurement aspect, polarization curves were obtained by using cyclic voltammetry (VoltaLab 50 potentiostat/galvanostat) at an operational temperature of $30^{\circ} \mathrm{C}$. The three-electrode configuration was employed in the circuit, with the sample as the working electrode, the graphite rod as the counter electrode, and the saturated calomel electrode (SCE) as the reference electrode. $3.5 \mathrm{wt} \%$ aqueous solution of saline was utilized as the electrolyte. Open circuit potential (OCP) at the equilibrium state of system was used as the corrosion potential ( $E_{\text {corr }}$ in [V] vs. SCE). For the potentiodynamic polarization experiments, the potential was scanned from -500 to $500 \mathrm{mV}$ at a scanning rate of $50 \mathrm{mV} \cdot \mathrm{s}^{-1}$. Corrosion current $\left(I_{\text {corr }}\left[\mu \mathrm{A} \cdot \mathrm{cm}^{-2}\right]\right)$ was determined by superimposing a straight line along the linear portion of the cathodic or anodic curve and extrapolating it through $E_{\text {corr }}[\mathrm{mV}]$. All raw data were taken at least three times to ensure the reproducibility and statistical significance.

\section{Results and discussion}

A representative process of base-catalyzed sol-gel reactions of MTMS and APTMS was used to prepare superhydrophobic MS microspheres. Moreover, the typical procedure for the preparation of biomimetic epoxy incorporated superhydrophobic MS microspheres coatings by replicating fresh Xanthosoma sagittifolium leaf through nanocasting technique was used, as shown in Figure 1. It should be noted that a large amount of MTMS in the sol-gel process may lead to as-prepared silica microspheres with a considerable amount of methyl groups attached to the surface, resulting in better superhydrophobicity. On the other hand, a small amount of APTMS used in the sol-gel process indicates that few primary amine groups may adhere to the surface of silica microspheres, implying the formation of chemical bonding between primary amine and the epoxide ring of epoxy pre-polymer.

\subsection{Characterization of superhydrophobic methyl-modified silica microspheres}

Figure 2 show the representative NMR, FTIR spectroscopy and SEM image of MS microspheres. Solidstate ${ }^{29} \mathrm{Si}$ NMR provided quantitative information about the condensation reaction and solid-state ${ }^{13} \mathrm{C}$ NMR spectroscopy was particularly useful in
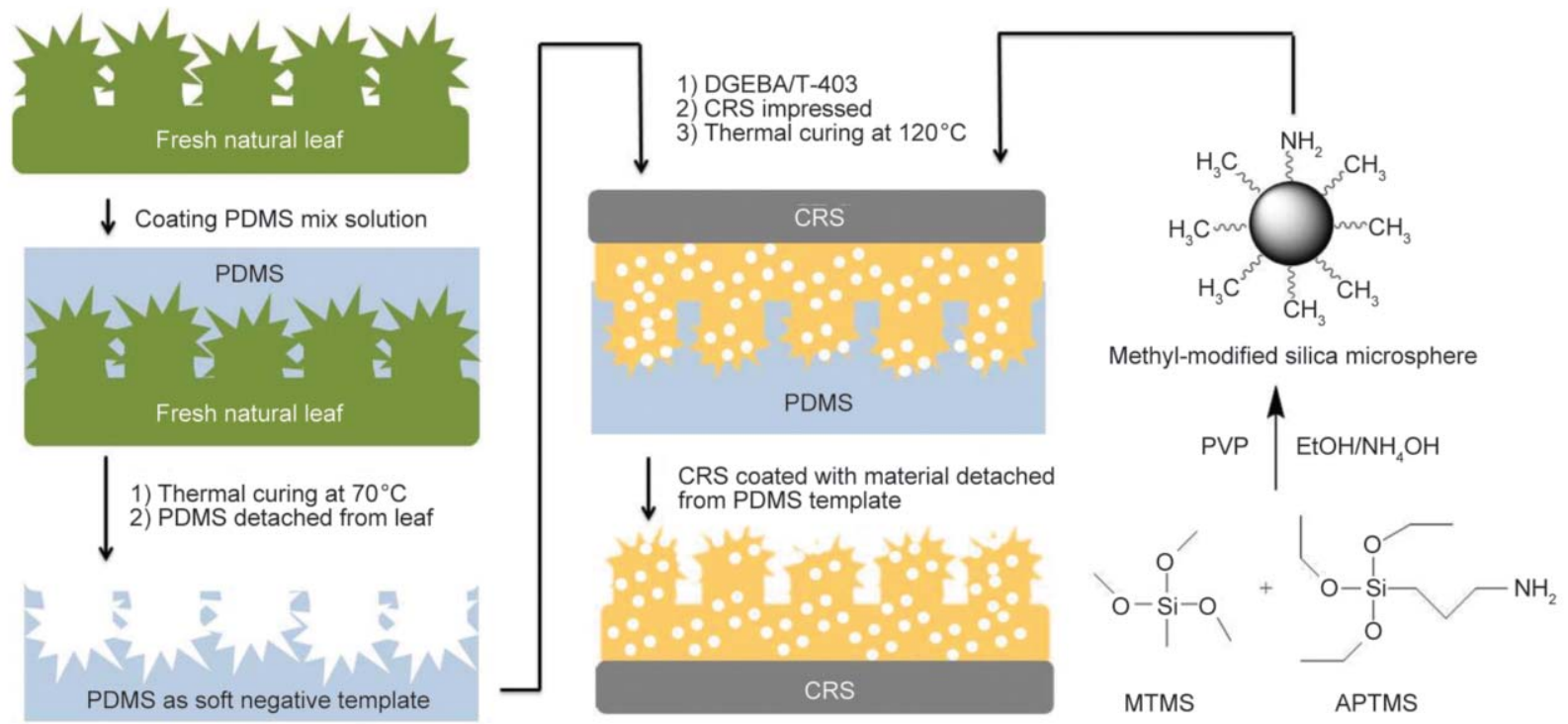

Figure 1. Preparation flow chart of superhydrophobic methyl-silica (MS) microsphere and incorporated superhydrophobic MS microspheres onto the biomimetic epoxy thermoset surface to manufacture the CRS coated with biomimetic epoxy thermoset composite 
monitoring the hydrolysis reaction of sol-gel process. Figure 2 a shows the ${ }^{29} \mathrm{Si} \mathrm{NMR}$ spectrum, two clear resonance peaks derived from $T_{\mathrm{m}}\left(T_{\mathrm{m}}=\right.$ $\mathrm{RSi}(\mathrm{OSi})_{\mathrm{m}}(\mathrm{OH})_{3-\mathrm{m}}, m=1-3 ; T_{3}$ at $\delta=-65.2 \mathrm{ppm}$ and $T_{2}$ at $\delta=-55.7 \mathrm{ppm}$ ) were observed [48]. $T_{1}$ peaks were too small to be analyzed in the photography. The formation of $T_{1}$ and $T_{0}$ species was insignificant, suggesting that non-reactive organically modified precursor was present. Figure $2 b$ shows the results of ${ }^{13} \mathrm{C}$ NMR analysis. The sample exhibited three peaks with nearly equal intensity at the position around 11.1, 26.1, and $43.5 \mathrm{ppm}$. Three peaks could be attributed to three different kinds of Carbon atoms for the APTMS group, confirming the existence of APTMS bonded to silica. Figure $2 \mathrm{c}$ displays the FTIR spectrum of MS microspheres. The presence of characteristic bands of MS at wavenumber of 774 and $2979 \mathrm{~cm}^{-1}$ were attributed to the stretching vibrations of $\mathrm{Si}-\mathrm{C}$ and $\mathrm{C}-\mathrm{H}$. The MS exhibited well-defined absorptions at ca. $1411 \mathrm{~cm}^{-1}$, which belonged to the characteristic of symmetric deformations in $\mathrm{Si}-\mathrm{R}$ groups. The presence of $\mathrm{Si}-\mathrm{O}-\mathrm{Si}$ linkages
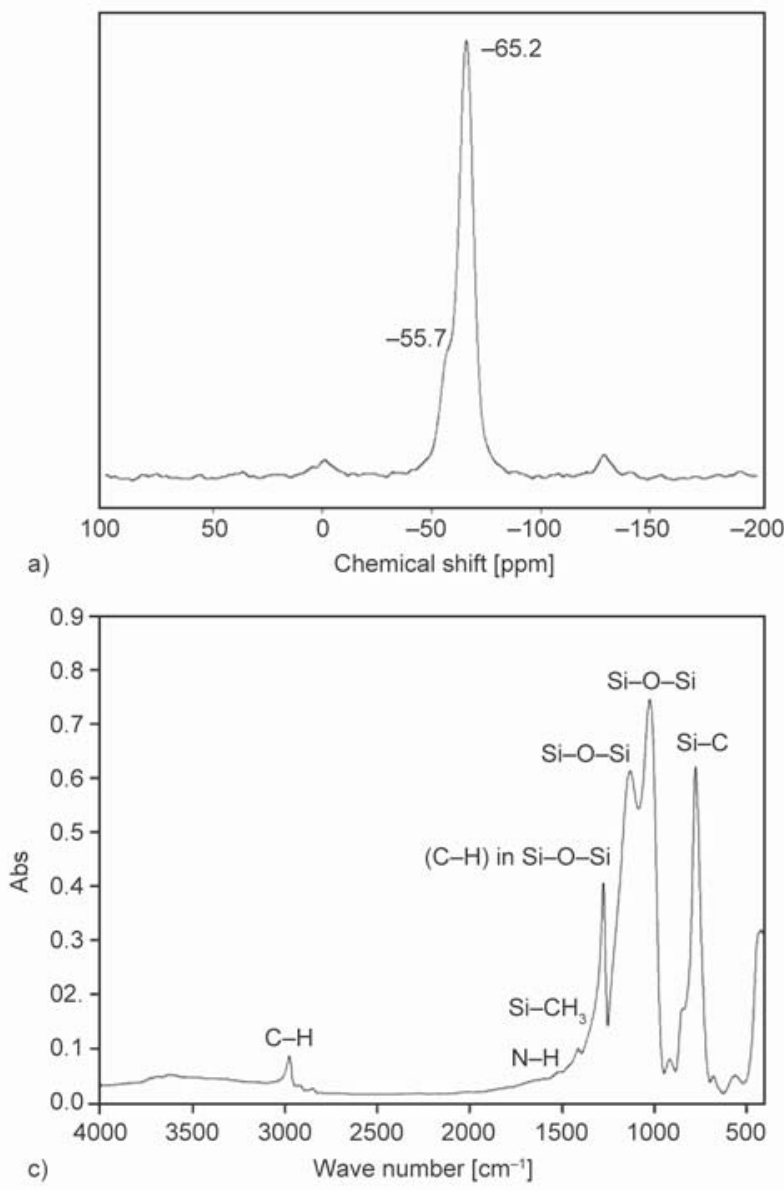

could be assigned to the absorptions at the location of 1136 and $1024 \mathrm{~cm}^{-1}$. Moreover, the band occurring at the location of $1273 \mathrm{~cm}^{-1}$ showed a well-defined $\mathrm{C}-\mathrm{H}$ absorption of $\mathrm{Si}-\mathrm{CH}_{3}$ [49]. Furthermore, the bond at $1531 \mathrm{~cm}^{-1}$ was due to $\mathrm{N}-\mathrm{H}$ bending vibration. Because the mole ratio of MTMS/APTMS in sol-gel reaction was 20 , meaning that the $\mathrm{Si}-\mathrm{CH}_{3}$ had a stronger absorption than $\mathrm{N}-\mathrm{H}$ in $\mathrm{MS}$, as shown in Figure 2c. Moreover, there were much more methyl groups providing the superhydrophobicity of the silica surface. Furthermore, the $\mathrm{N}-\mathrm{H}$ group could provide the strong chemical bonding with epoxy. The Figure $2 \mathrm{~d}$ shows the SEM image of the as-prepared MS microspheres at 10000 magnification, revealing an average particle size of $\sim 1 \mu \mathrm{m}$ in diameter.

\subsection{Investigating the thermal curing of epoxy thermosets by FTIR Spectroscopy and XPS analysis}

FTIR was utilized to investigate the curing process of the epoxy thermoset. For example, FTIR spectra of epoxy slurry (before curing), biomimetic epoxy ther-

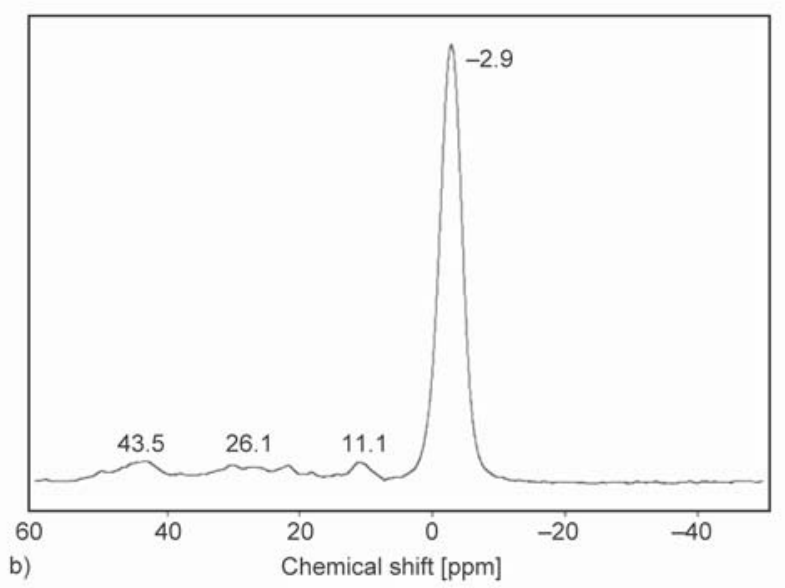

b)

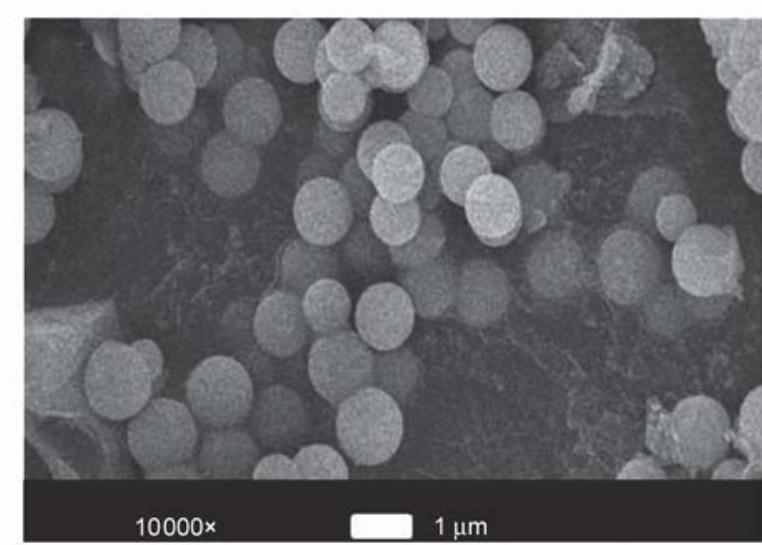

d)

Figure 2. The identification of as-prepared superhydrophobic methyl-silica microsphere of (a) ${ }^{29} \mathrm{Si}$ NMR spectroscopy, (b) ${ }^{13}$ C NMR spectroscopy, (c) FTIR spectroscopy and (d) image of SEM observation at magnification $10000 \times$ 
moset (after curing) and biomimetic epoxy-silica composite are shown in Figure 3. After the programmed heat treatment, the disappearance of characteristic peak of epoxide ring $\left(915 \mathrm{~cm}^{-1}\right)$ and of the primary amine of epoxy thermoset could be identified as a proof of the accomplishment of the ringopening polymerization, as shown in Figures $3 b$ and 3c. Moreover, XPS was utilized to measure the characteristic peak of BET and BEC, as shown in Figure 4 . From the Figure $4 b$, the Si $2 p$ and Si s were found to be locatet at the positions of $102.9 \mathrm{eV}(\mathrm{Si}-\mathrm{O}$ group) and $153.5 \mathrm{eV}$, indicating that the MS microspheres were randomly dispersed onto the BEC surface.

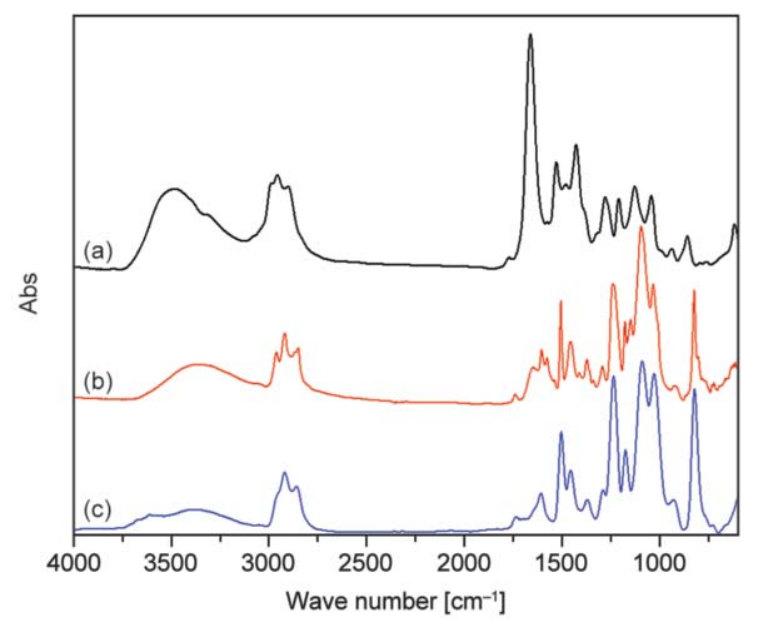

Figure 3. The identification of FTIR spectroscopy for (a) epoxy slurry (before curing), (b) epoxy thermoset (after curing) and (c) composite with biomimetic epoxy thermoset and incorporated superhydrophobic MS microspheres

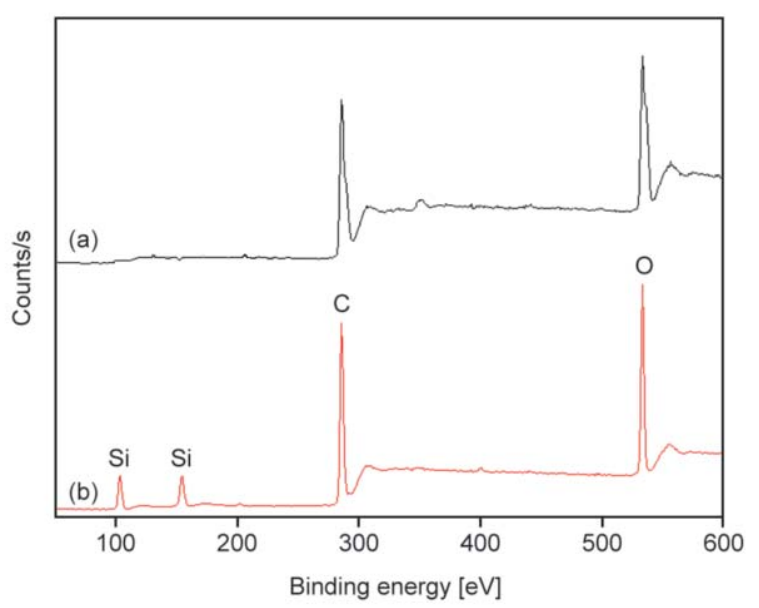

Figure 4. The identification of XPS for (a) biomimetic epoxy thermoset and (b) composite with biomimetic epoxy thermoset and incorporated superhydrophobic MS microspheres

\subsection{Microscopic observations and EDX spectroscopy for biomimetic morphology}

Macroscopic photograph of natural, fresh leaf of Xanthosoma sagittifolium is shown in Figure 5a. Moreover, microscopic SEM overview image at a magnification of $1000 \times$ for Figure $5 b$ fresh natural leaf, Figure 5c PDMS soft negative template, Figure 5d biomimetic epoxy thermoset and Figure 5e composite with synergistic effect of biomimetic epoxy thermoset morphology and incorporated superhydrophobic MS microspheres. In Figure 5b, there were much smaller micro papillary hills and nano textures tan those found on the fresh natural Xanthosoma sagittifolium leaves. Moreover, the Figure 5c shows the SEM image of the cured PDMS negative template prepared by casting the liquid PDMS directly onto a natural and fresh Xanthosoma sagittifolium leaves. The Figure 5d was the SEM image of BET at magnification of $1000 \times$. It should be noted that the morphology of BET is almost the same as that of fresh Xanthosoma sagittifolium leaves, implying that the nano-casting technique was successful in transferring the pattern of natural leaves. To well-understand the papilla-like and texture on the surface, Figure $5 \mathrm{e}$ shows the SEM image of BET at a magnification of $3000 \times$. From the cross-section Figure $5 \mathrm{f}$ at a magnification of 5000×, the height of papilla-like was found to be $6-9 \mu \mathrm{m}$. This observation depicted that the template effectively replicated the structure of leaf surface.

For the morphology study of biomimetic epoxy silica containing $3 \mathrm{wt} \%$ composite (BEC-3\%), the SEM image at a magnification of $1000 \times$ of BEC- $3 \%$ images, Si mapping and EDX of the BEC-3\% surface are presented in Figures 6. Morphology of BEC-3\% was very similar to that of BET, implying that the majority of incorporated MS microspheres may be embedded into the BET matrix, as shown in Figure 6a. In Figure 6b, the lots of red spots representing the mapping of Si element of BEC was clearly found, indicating that the MS microspheres were randomly dispersed in the BET matrix. Moreover, the EDX showed that the appearance of peaks for $\mathrm{Si}, \mathrm{O}$ and $\mathrm{N}$ element of BEC-3\%, implying that the MS microspheres may probably be there inside or on the BET coating. Hydrophobicity enhancement of BEC-3\% coating resulted from both the biomimetic morphol- 


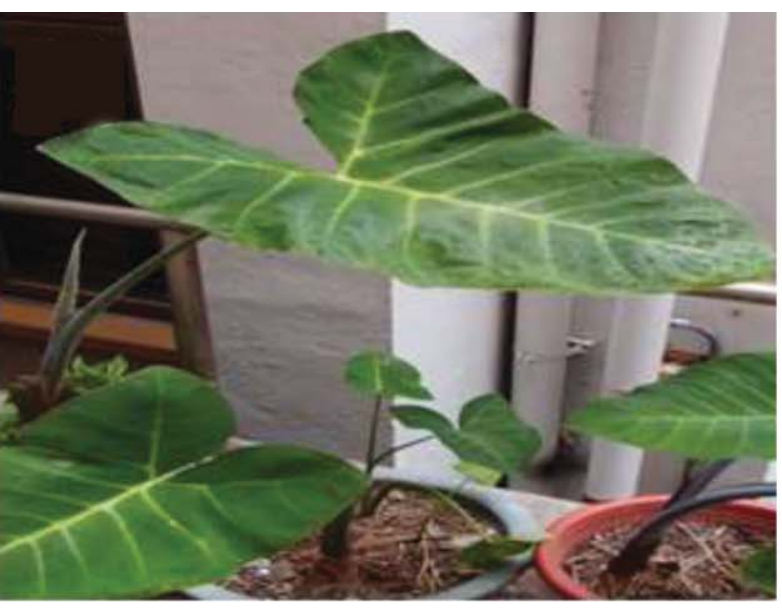

a)

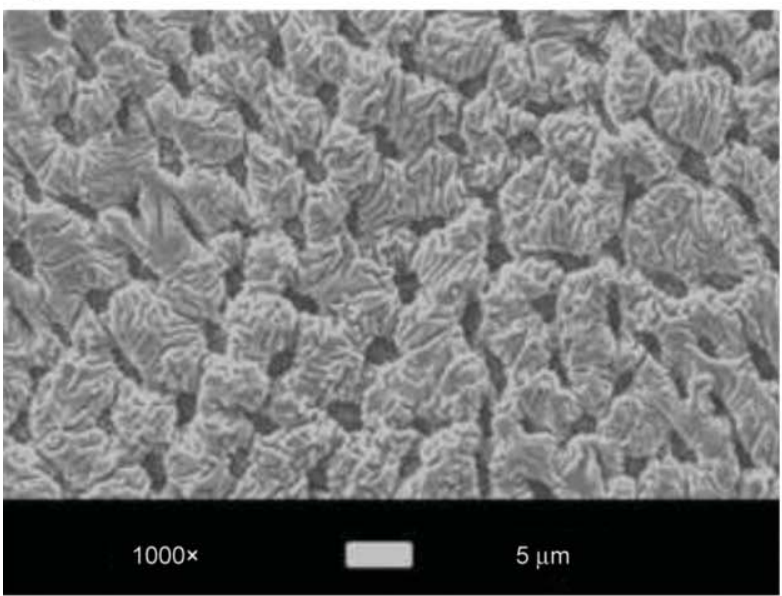

c)

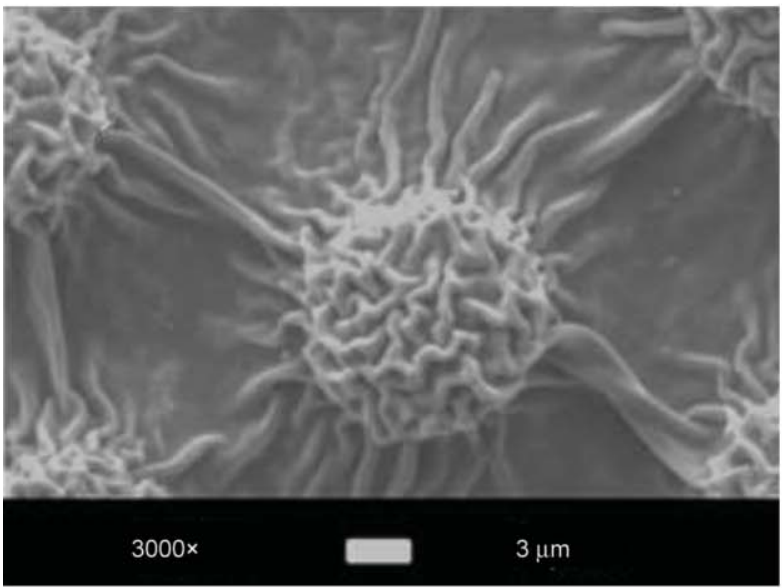

e)

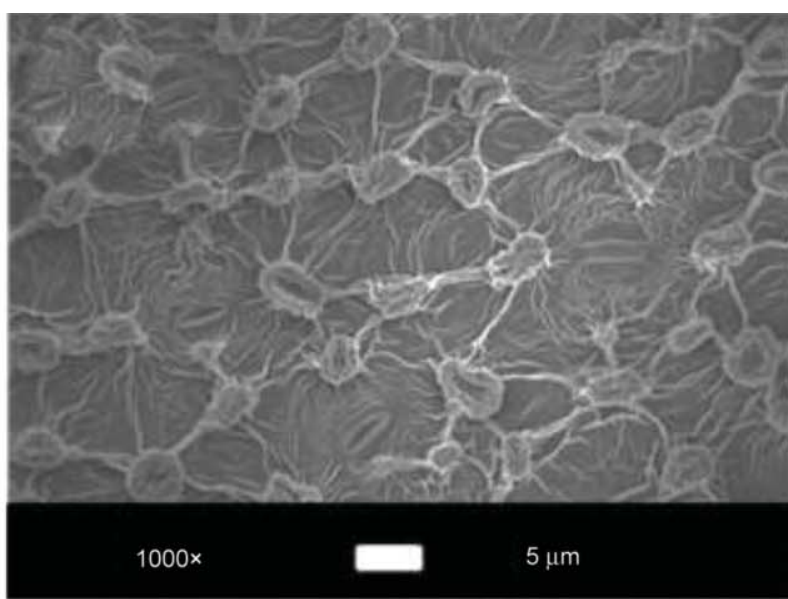

b)

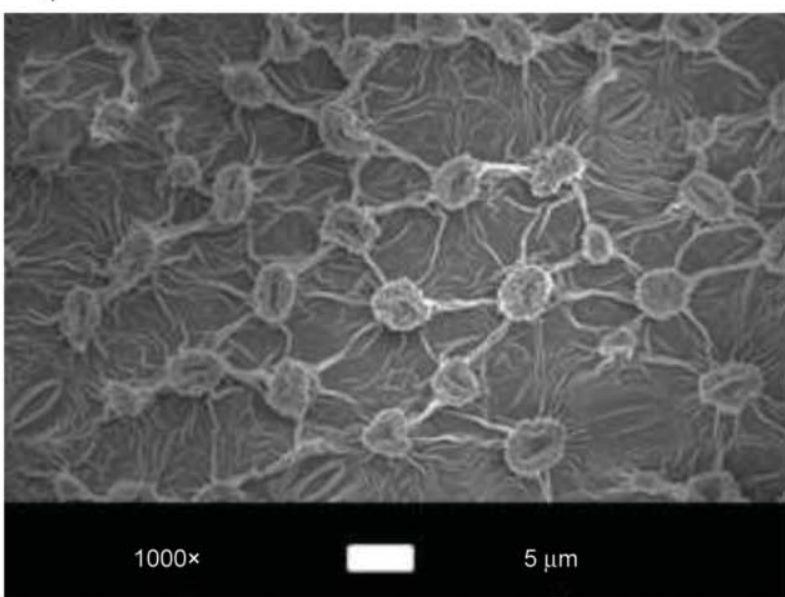

d)

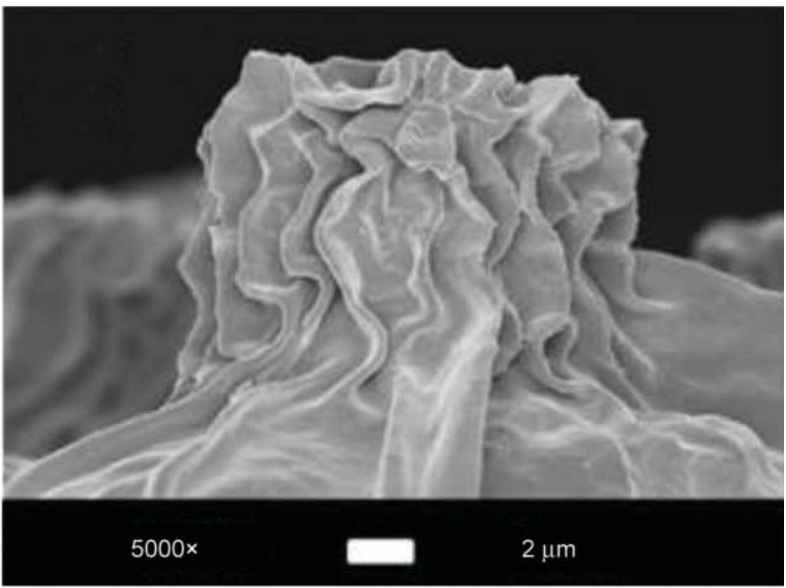

f)

Figure 5. The morphology observations for (a) macroscopoc photograph of the fresh natural Xanthosoma sagittifolium leaves. Microscopic SEM overlook image at magnification of 1000× for (b) fresh natural leaf, (c) PDMS soft negative template, (d) biomimetic epoxy thermoset and (e) biomimetic epoxy thermoset at magnification of $3000 \times(\mathrm{f})$ crosssection for biomimetic epoxy thermoset at magnification of $5000 \times$

ogy and incorporated MS microspheres, respectively, can be further identified by the contact angle measurement of water droplets and will be discussed in the following section.

\subsection{Atomic force microscope}

In Figure 7, the three dimensional AFM images of biomimetic epoxy thermoset and biomimetic epoxy silica containing $3 \mathrm{wt} \%$ composite on the CRS sub- 


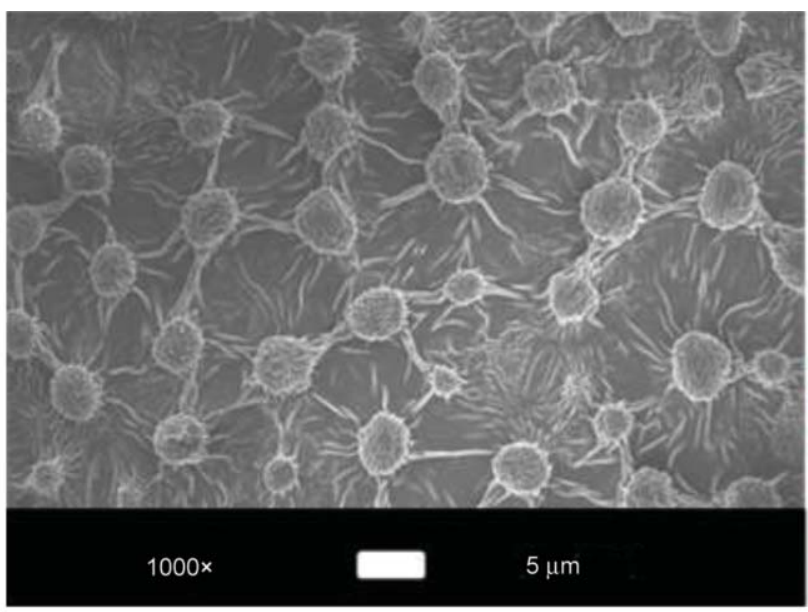

a)

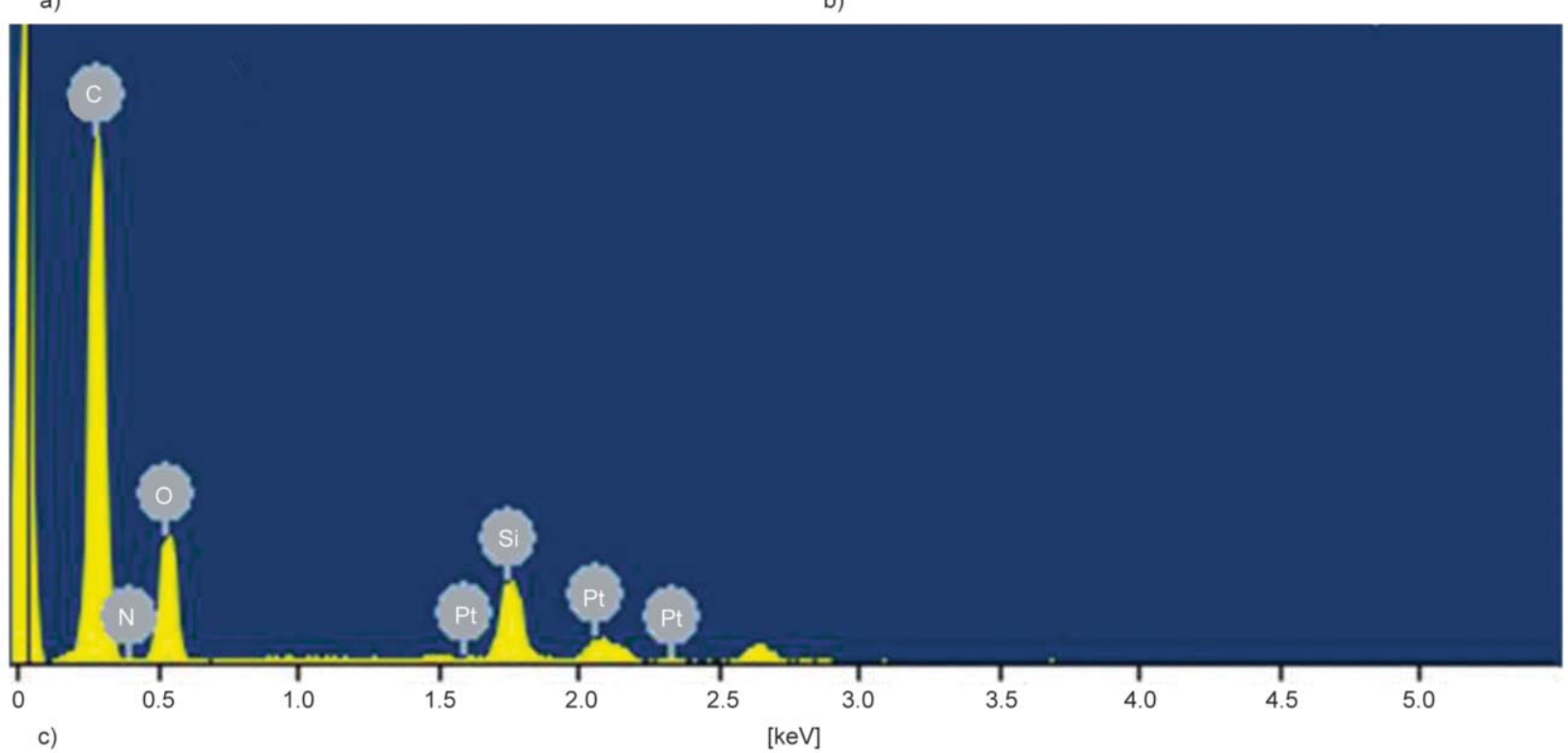

Figure 6. The surface studies of as-prepared biomimetic epoxy-silica composite of (a) SEM microscopic observation at magnification of $1000 \times$, (b) Si mapping and (c) energy-dispersive X-ray spectroscopy

strate vividly showed higher surface roughness. From the AFM image, the surface morphology was coincident with the SEM observation. Moreover, wellunderstanding the surface roughness on the BET and BEC coatings, AFM was studied and performed in which height images were obtained and average

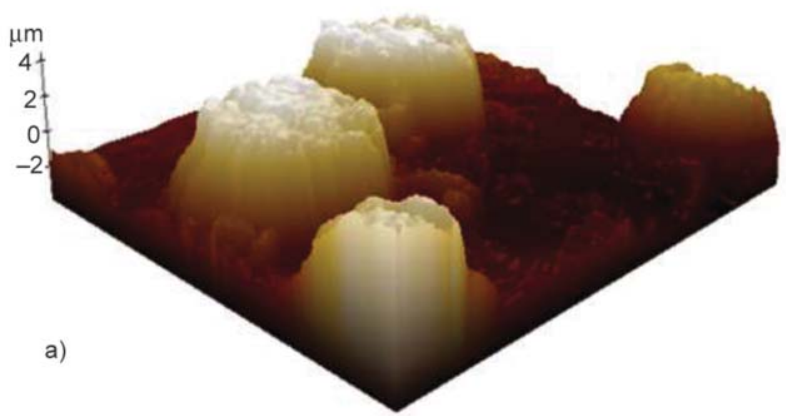

roughness levels of the surfaces were determined [50]. The arithmetic average roughness, Ra levels of the BET and BEC-3\% coatings were measured. The BEC-3\% coating had the higher RMS roughness of $2.322 \mu \mathrm{m}$ than BET coating $(2.205 \mu \mathrm{m})$.

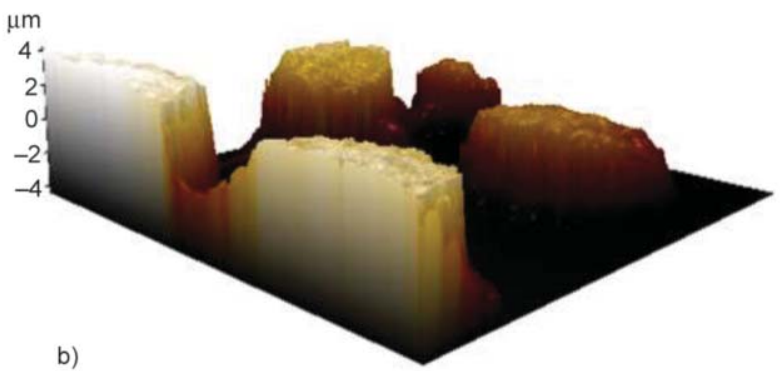

Figure 7. The surface roughness studies of three dimonsional morphology atomic force microscope for (a) CRS electrode coated with biomimetic epoxy thermoset and (b) CRS electrode coated with biomimetic epoxy-silica composite 


\subsection{Surface hydrophobicity of as-prepared coatings measured by contact angle}

In this study, the contact angle (CA) of water droplets upon six different surfaces of coatings were illustrated in Figure 8. The CA for fresh leaf of Xanthosoma sagittifolium was $146^{\circ}$, as shown in Figure 8a. The CA for neat epoxy thermoset with smooth surface was $81^{\circ}$, as shown in Figure $8 \mathrm{~b}$. Moreover, the CA upon surface of BET was $123^{\circ}$, as shown in Figure 8c. It indicated clearly that the biomimetic morphology may increase the CA of neat epoxy thermoset by up to $42^{\circ}$, reflecting that the biomimetic morphology could effectively promote the hydrophobicity of neat material. Moreover, the CA of neat as-prepared dry fine powder coating of superhydrophobic MS microspheres on glass was found to be $158^{\circ}$ because of the methyl group attached onto the surface, as shown in Figure $8 d$. With incorporated $1 \mathrm{wt} \%$ of MS microspheres into BET, the as-obtained BEC-1\% with synergistic effect of biomimetic morphology and superhydrophobic silica microspheres was found to exhibit the $\mathrm{CA}=137^{\circ}$, as shown in Figure 8e. Moreover, incorporated $3 \mathrm{wt} \%$ of MS microspheres into BET, the as-perpared $\mathrm{BEC}-3 \%$ was revealed the $\mathrm{CA}=153^{\circ}$, as shown in Figure 8e. It indicated that introducing $3 \mathrm{wt} \%$ silica microspheres into BET coating may further promote the hydrophobicity of BET by further increasing the CA of coating by $\sim 30^{\circ}$. In summary, the BEC coating with synergistic effect of biomimetic epoxy thermoset morphology and incorporated MS microspheres revealed an increment in CA of $72^{\circ}$ as compared to that of neat epoxy thermoset with smooth surface, implying that this composite could be a potential candidate as corrosion protection coating, as discussed in the following section.

\subsection{Potentiodynamic measurements}

Polarization curves for the CRS electrode coated with neat epoxy thermoset, biomimetic epoxy thermoset and biomimetic epoxy-silica composite can be obtained by performing all the measurements in electrolyte of $3.5 \mathrm{wt} \% \mathrm{NaCl}$ aqueous solution.

Corrosion current was obtained by extrapolating Tafel plots, from both the cathodic and anodic polarization curves for the respective corrosion process. Extrapolating the cathodic and anodic polarization curves to the point of intersection provides both the corrosion potential and corrosion current. Tafel plots for (a) bare CRS electrode, (b) CRS electrode coated with smooth epoxy thermoset, (c) CRS electrode coated with biomimetic epoxy thermoset, (d) CRS electrode coated with biomimetic epoxy-silica containing $1 \mathrm{wt} \%$ composite and (e) CRS electrode coated with biomimetic epoxy-silica containing $3 \mathrm{wt} \%$ com-

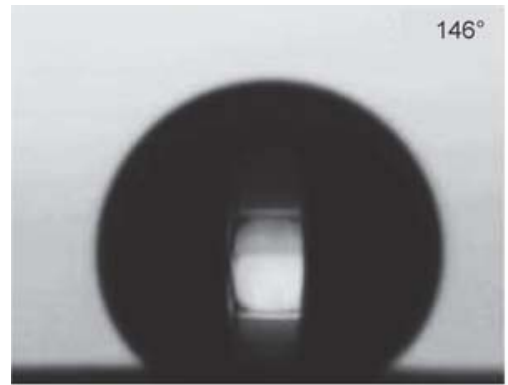

a)

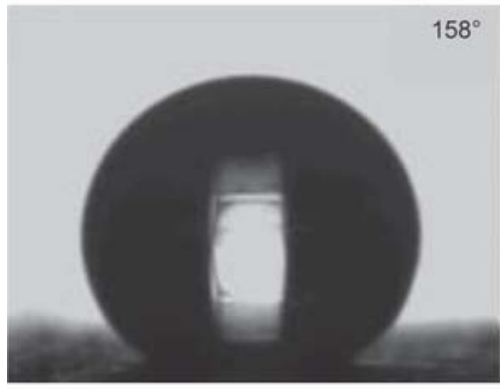

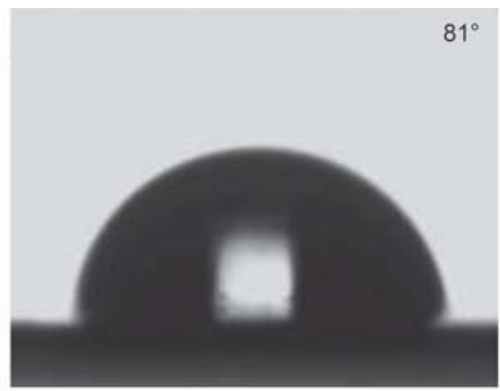

b)

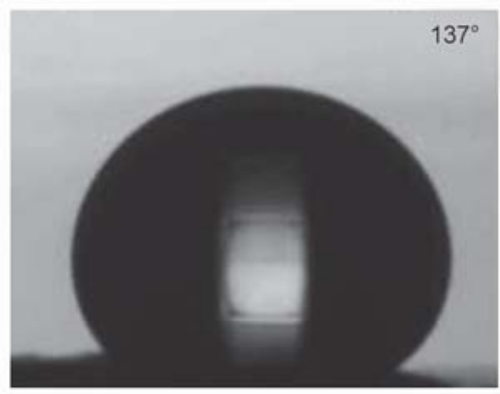

e)

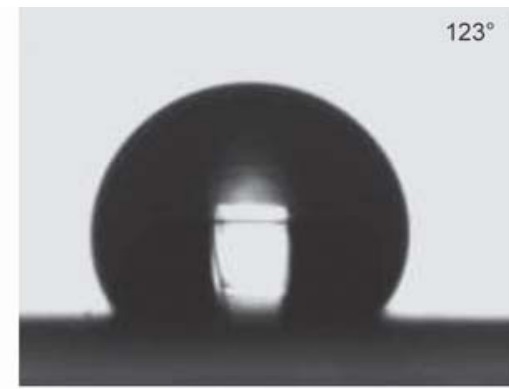

c)

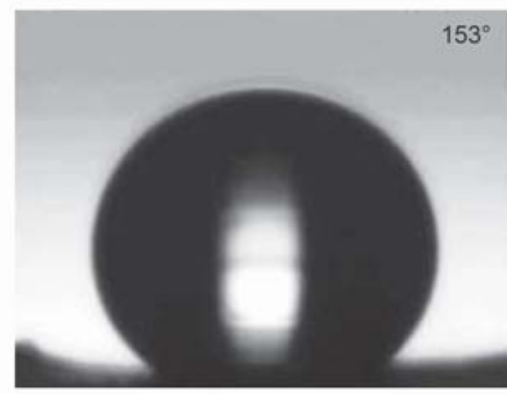

f)

Figure 8. Water contact angle for (a) the fresh natural Xanthosoma sagittifolium leaves (b) RS electrode coated with smooth epoxy thermoset, (c) CRS electrode coated with biomimetic epoxy thermoset, (d) methyl-silica microsphere tablet, (e) CRS electrode coated with biomimetic epoxy-silica containing 1\% composite and (f) CRS electrode coated with biomimetic epoxy-silica containing 3\% composite 


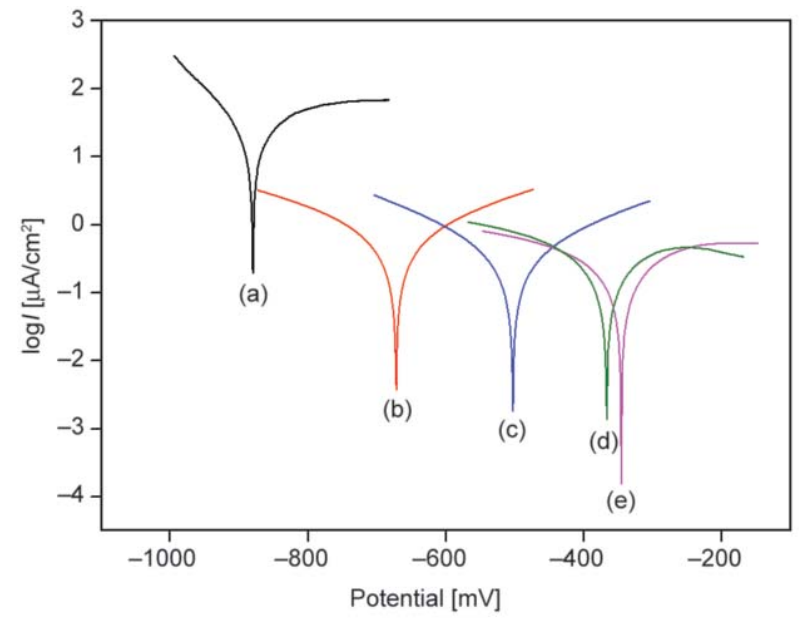

Figure 9. Tafel plots of electrochemical corrosion measurement for (a) bare CRS electrode, (b) CRS electrode coated with smooth epoxy thermoset, (c) CRS electrode coated with biomimetic epoxy thermoset, (d) CRS electrode coated with biomimetic epoxy-silica containing 1\% composite and (e) CRS electrode coated with biomimetic epoxy-silica containing $3 \%$ composite

posite are shown in Figure 9 and the corresponding data were summarized and listed in Table 1.

Tafel plots of CRS electrode coated with all three samples exhibiting the corrosion potential $\left(E_{\text {corr }}\right)$ was more positive than the bare CRS electrode. On the other hand, the corrosion current $\left(I_{\text {corr }}\right)$ of the CRS electrode coated with samples was found to be lower than the bare CRS electrode. First of all, studies for the effect of biomimetic epoxy morphology on corresponding corrosion protection upon CRS electrode, it should be noted that the corrosion potential of CRS electrode coated with BET $\left(E_{\text {corr }}=-504.0 \mathrm{mV}\right)$ was found to be more positive than that of neat epoxy thermoset $\left(E_{\text {corr }}=-672.5 \mathrm{mV}\right)$. Moreover, the corrosion current of CRS electrode coated with BET ( $\left.I_{\text {corr }}=0.33 \mu \mathrm{A} / \mathrm{cm}^{2}\right)$ was found to be lower than that of neat epoxy thermoset $\left(I_{\text {corr }}=0.78 \mu \mathrm{A} / \mathrm{cm}^{2}\right)$. Based

Table 1. Contact angle and electrochemical corrosion measurements of bare CRS electrode, CRS coated with smooth epoxy thermoset, BET and BEC

\begin{tabular}{|c|c|c|c|c|c|}
\hline \multirow[b]{2}{*}{$\begin{array}{l}\text { Sample } \\
\text { code }\end{array}$} & \multicolumn{5}{|c|}{ Electrochemical corrosion measurements } \\
\hline & $\begin{array}{l}E_{\text {corr }} \\
{[\mathrm{mV}]}\end{array}$ & $\begin{array}{c}I_{\text {corr }} \\
{\left[\mu \mathrm{A} / \mathbf{c m}^{2}\right]}\end{array}$ & $\begin{array}{c}\text { Contact } \\
\text { angle } \\
{\left[{ }^{\circ}\right]}\end{array}$ & Hysteresis & $\begin{array}{c}\text { Coating } \\
\text { thickness } \\
{[\mu \mathrm{m}]}\end{array}$ \\
\hline CRS & -852.8 & 220.5 & - & - & - \\
\hline Epoxy & -672.5 & 0.78 & $81 \pm 3$ & 32 & $28 \pm 2$ \\
\hline BET & -504.0 & 0.33 & $123 \pm 3$ & 21 & $29 \pm 2$ \\
\hline BEC-1\% & -387.2 & 0.31 & $137 \pm 3$ & 17 & $30 \pm 2$ \\
\hline BEC-3\% & -344.4 & 0.19 & $153 \pm 2$ & 9 & $31 \pm 2$ \\
\hline
\end{tabular}

on the $E_{\text {corr }}$ and $I_{\text {corr }}$ data obtained from Tafel plots, the BET was found to show better corrosion protection upon CRS electrode than that of epoxy thermoset with smooth surface. The increase of anticorrosion performance of the coating may be attributed to the more hydrophobic surface $\left(\mathrm{CA}=123^{\circ}\right)$ of BET than that of neat epoxy thermoset $\left(\mathrm{CA}=81^{\circ}\right)$. Secondly, the further studies for the effect of incorporated superhydrophobic $1 \mathrm{wt} \% \mathrm{MS}$ microspheres existing in BET on corrosion protection upon CRS electrode, it should be noted that the corrosion potential of CRS electrode coated with BEC-1\% $\left(E_{\text {corr }}=-387.2 \mathrm{mV}\right)$ was found to be more positive than that of BET $\left(E_{\text {corr }}=-504.0 \mathrm{mV}\right)$. Moreover, the corrosion current of CRS electrode coated with BEC- $1 \%\left(I_{\text {corr }}=0.31 \mu \mathrm{A} / \mathrm{cm}^{2}\right)$ was found to be lower than that of BET $\left(I_{\text {corr }}=0.33 \mu \mathrm{A} / \mathrm{cm}^{2}\right)$. Afterward, incorporated superhydrophobic $3 \mathrm{wt} \%$ MS microspheres existing in BET on corrosion protection upon CRS electrode, it described that the corrosion potential of CRS electrode coated with BEC-3\% $\left(E_{\text {corr }}=-344.4 \mathrm{mV}\right)$ was found to be more positive than that of BEC-1\% $\left(E_{\text {corr }}=-387.2 \mathrm{mV}\right)$. Moreover, the corrosion current of CRS electrode coated with BEC-3\% $\left(I_{\text {corr }}=0.19 \mu \mathrm{A} / \mathrm{cm}^{2}\right)$ was found to be lower than that of BET and BEC-1\% ( $I_{\text {corr }}=$ $0.31 \mu \mathrm{A} / \mathrm{cm}^{2}$ ). The BEC-3\% was found to show better corrosion protection upon CRS electrode than that of BET and BEC- $1 \%$ based on the conclusion obtained from the $E_{\text {corr }}$ and $I_{\text {corr }}$ data. The further increase of anticorrosion performance of coating may be associated with incorporated suitable amount MS microspheres enhance the hydrophobicity of BET and BEC- $1 \%$ coating.

Enhancement of coatings in anticorrosion upon CRS electrode can be further confirmed by electrochemical impedance spectroscopy (EIS), as discussed in the following section.

\subsection{Electrochemical impedance spectroscopy measurements}

In this study, EIS was utilized to evaluate the corrosion protection for CRS electrode coated with epoxy thermoset, BET and BEC. Generally, corrosion behavior of a metal can be modeled with an equivalent circuit which consists of a double-layered capacitor that is parallel with a charge transfer resistor and connected in series with an electrolyte solution re- 
sistor. Impedance $(Z)$ depends on the charge transfer resistance $\left(R_{\mathrm{ct}}\right)$, the solution resistance $\left(R_{\mathrm{s}}\right)$, the capacitance of the electrical double layer, and the frequency of the AC signal $(\omega)$.

The high-frequency intercept was equal to the solution resistance and the low-frequency intercept was equal to the sum of the solution and charge transfer resistances [51]. Figure 10 shows the Nyquist plots for (a) bare CRS electrode, (b) CRS electrode coated with smooth epoxy thermoset, (c) CRS electrode coated with biomimetic epoxy thermoset, (d) CRS electrode coated with biomimetic epoxy-silica containing $1 \%$ composite and (e) CRS electrode coated with biomimetic epoxy-silica containing 3\% composite. The samples were immersed in $3.5 \mathrm{wt} \% \mathrm{NaCl}$ aqueous electrolyte for $40 \mathrm{~min}$ before EIS measurements. The charge transfer resistances of all samples, as determined by subtracting the intersection of the highfrequency end from the low-frequency end of the semi-circle arc with the real axis, were $0.156,574.8$, 3140,3961 and $5250 \mathrm{k} \Omega \cdot \mathrm{cm}^{2}$, respectively.

EIS Bode plots (impedance vs. frequency) of all samples were shown in Figure 11. $Z_{\text {real }}$ was also a measure of corrosion resistance. Low $Z_{\text {real }}$ value could be performed from high capacitance to low resistance of the coating. The Bode magnitude plots for (a) bare CRS electrode, (b) CRS electrode coated with smooth epoxy thermoset, (c) CRS electrode coated with biomimetic epoxy thermoset, (d) CRS electrode coated

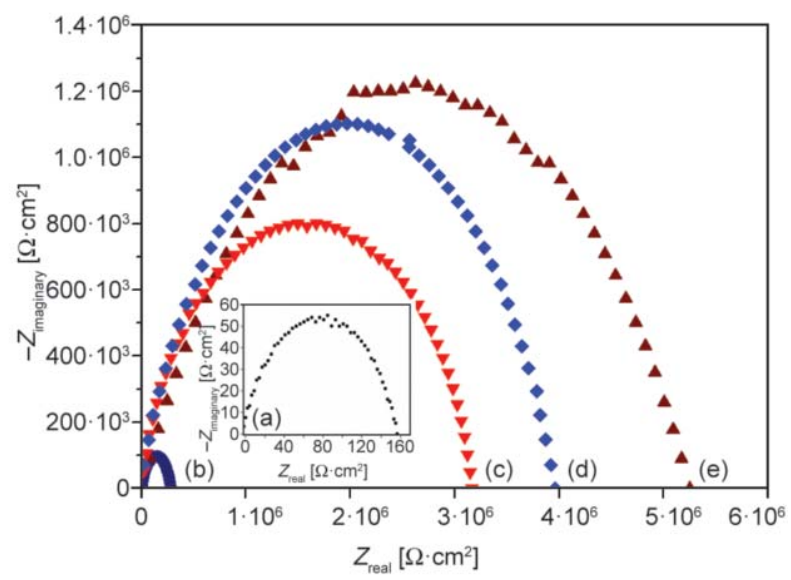

Figure 10. Nyquist plots of electrochemical corrosion measurement for (a) bare CRS electrode, (b) CRS electrode coated with smooth epoxy thermoset, (c) CRS electrode coated with biomimetic epoxy thermoset, (d) CRS electrode coated with biomimetic epoxy-silica containing $1 \%$ composite and (e) CRS electrode coated with biomimetic epoxy-silica containing 3\% composite

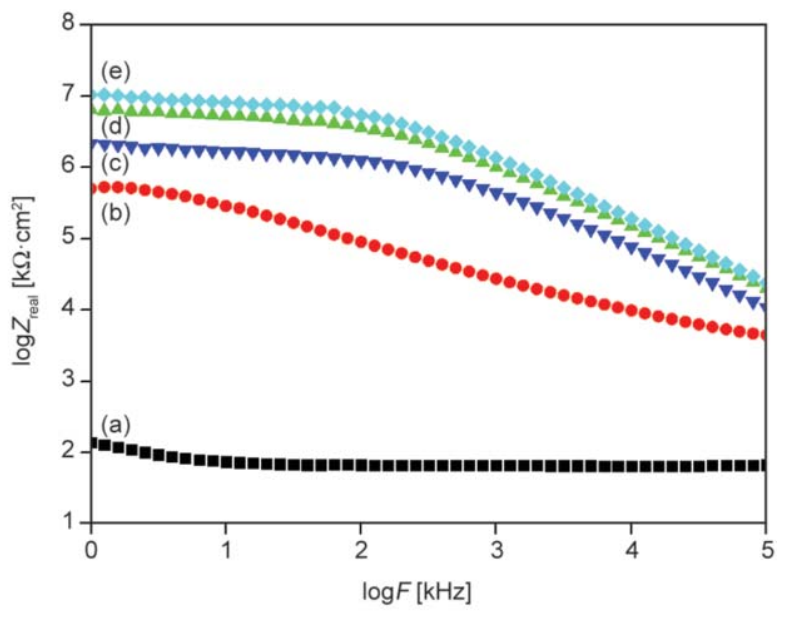

Figure 11. Bode plots of electrochemical corrosion measurement for (a) bare CRS electrode, (b) CRS electrode coated with smooth epoxy thermoset, (c) CRS electrode coated with biomimetic epoxy thermoset, (d) CRS electrode coated with biomimetic epoxy-silica containing $1 \%$ composite and (e) CRS electrode coated with biomimetic epoxy-silica containing 3\% composite

with biomimetic epoxy-silica containing $1 \%$ composite and (e) CRS electrode coated with biomimetic epoxy-silica containing 3\% composite showed $Z_{\text {real }}$ values of $2.104,5.724,6.333,6.814$ and $7.017 \mathrm{k} \Omega \cdot \mathrm{cm}^{2}$, respectively, at low frequency end. It should be noted that the result obtained from the EIS (Nyquist plots and Bode plots) was found to be consistent with the previous conclusion obtained from the Tafel plots. It also demonstrated that the composite with synergistic effect of biomimetic epoxy thermoset morphology and incorporated MS microspheres exhibiting better corrosion protection as compared to that of neat epoxy thermoset with smooth surface.

In this work, the superhydropobic composite coatings with synergistic effect of biomimetic epoxy thermoset morphology and incorporated MS microspheres had the best corrosion protection. The possible mechanism of enhancing corrosive protection prop-

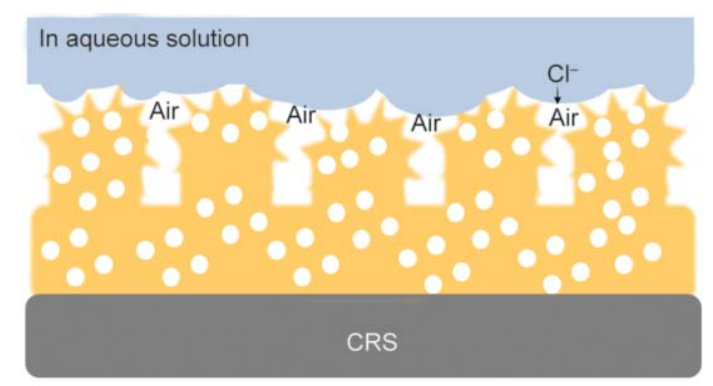

Figure 12. The mechanism of enhancing corrosive protection properties 
erties was that superhydrophobic surface composed of hills trapped the gas within the valleys between the hills. Therefore, the free radical, $\mathrm{O}_{2}$ and $\mathrm{H}_{2} \mathrm{O}$ in the corrosion solution could not effectively separate and reach to metal substrates, as shown in Figure 12.

\section{Conclusions}

In this work, the coating of composite with synergistic effect of biomimetc epoxy thermoset morphology and incorporated superhydrophobic MS microspheres was found to reveal better anticorrosion performance on CRS electrode based on a series of electrochemical measurements (Tafel plots) in saline conditions. First of all, MS microspheres were prepared by performing the sol-gel reaction of MTMS in the presence of APTMS, followed by characterized through FTIR, ${ }^{13} \mathrm{C}$ NMR and ${ }^{29} \mathrm{Si}$ solid-state NMR spectroscopy. The PDMS was employed as negative soft template to duplicate the surface structures of natural leaf of Xanthosoma sagittifolium. Moreover, the coatings with surface of biomimetic epoxy thermoset and biomimetic epoxy-silica composite were prepared by pouring the corresponding epoxy and epoxy-silica slurry upon PDMS template, followed by a series of programmed heating treatment. The surface morphology study of coatings with biomimetic morphology was investigated by the SEM observations. The distribution of MS microspheres in BEC-3\% coatings was identified by Si mapping and EDX studies, implying that the majority of MS microsphere was embedded in the BET coating. Moreover, the surface hydrophobicity of coatings with/ without biomimetic morphology and with MS microspheres was confirmed by the measurements of contact angle (CA) of water droplets. It clearly indicated that the contact angle of BEC-3\% $\left(\mathrm{CA}=153^{\circ}\right)$ and $\operatorname{BET}\left(\mathrm{CA}=123^{\circ}\right)$ was found to be much higher than that of neat epoxy thermoset $\left(\mathrm{CA}=81^{\circ}\right)$. The CRS electrode coated with BET was found to exhibit better anticorrosion performance than that of neat epoxy thermoset based on a series of electrochemical measurements. This may be attributed to the higher surface hydrophobicity of BET, resulted from the biomimetic morphology, than that of neat epoxy thermoset. Moreover, the CRS electrode coated with BEC-3\% was found to reveal better anticorrosion performance than that of BET. This may be related to the incorporated $3 \mathrm{wt} \%$ of superhydrophobic MS microspheres into BET to enhance the hydrophobicity of coating. In summary, the composite coating with synergistic effect of biomimetic epoxy thermoset morphology and incorporated superhydrophobic MS microspheres upon CRS electrode revealed significantly increased corrosion protection performance as compared to that of neat epoxy thermoset with smooth surface.

\section{Acknowledgements}

The authors acknowledge financial support from the Ministry of Science and Technology, Taiwan, R.O.C. (NSC 102-2632M-033-001-MY3) and (MOST 104-2113-M-033-001-MY3); the Department of Chemistry at CYCU; and the Center for Nanotechnology and Center for Biomedical Technology at CYCU.

\section{References}

[1] Newman R. C., Sieradzki K.: Metallic corrosion. Science, 263, 1706-1709 (1994). DOI: $10.1126 /$ science. 263.5154 .1708

[2] Ramezanzadeh B., Attar M. M., Farzam M.: A study on the anticorrosion performance of the epoxy-polyamide nanocomposites containing $\mathrm{ZnO}$ nanoparticles. Progress in Organic Coatings, 72, 410-422 (2011).

DOI: $10.1016 /$ j.porgcoat.2011.05.014

[3] Arefinia R., Shojaei A., Shariatpanahi H., Neshati J.: Anticorrosion properties of smart coating based on polyaniline nanoparticles/epoxy-ester system. Progress in Organic Coatings, 75, 502-508 (2012).

DOI: $10.1016 /$ j.porgcoat.2012.06.003

[4] Yeh J-M., Hsieh C-F., Jaw J-H., Kuo T-H., Huang H-Y., Lin C-L., Hsu M-Y.: Organo-soluble polyimde (ODABSAA)/montmorillonite nanocomposite materials prepared by solution dispersion technique. Journal of Applied Polymer Science, 95, 1082-1090 (2005). DOI: 10.1002/app.21317

[5] Ramezanzadeh B., Attar M. M.: An evaluation of the corrosion resistance and adhesion properties of an epoxy-nanocomposite on a hot-dip galvanized steel (HDG) treated by different kinds of conversion coatings. Surface and Coatings Technology, 205, 4649-4657 (2011). DOI: $10.1016 /$ j.surfcoat.2011.04.001

[6] Yan X., Xu G.: Corrosion and mechanical properties of epoxy-polyurethane/bronze composite coatings with low infrared emissivity. Surface and Coatings Technology, 205, 2307-2312 (2010).

DOI: $10.1016 /$ j.surfcoat.2010.09.015

[7] Gurunathan T., Rao C. R. K., Narayan R., Raju K. V. S. N.: Synthesis, characterization and corrosion evaluation on new cationomeric polyurethane water dispersions and their polyaniline composites. Progress in Organic Coatings, 76, 639-647 (2013). DOI: 10.1016/j.porgcoat.2012.12.009 
[8] Yu Y-H., Yeh J-M., Liou S-J., Chen C-L., Liaw D-J., Lu H-Y.: Preparation and properties of polyimide-clay nanocomposite materials for anticorrosion application. Journal of Applied Polymer Science, 92, 3573-3582 (2004).

DOI: $10.1002 /$ app. 20400

[9] Huttunen-Saarivirta E., Yudin V. E., Myagkova L. A., Svetlichnyi V. M.: Corrosion protection of galvanized steel by polyimide coatings: EIS and SEM investigations. Progress in Organic Coatings, 72, 269-278 (2011). DOI: 10.1016/j.porgcoat.2011.04.015

[10] Yeh J-M., Liou S-J., Lai C-Y., Wu P-C., Tsai T-Y.: Enhancement of corrosion protection effect in polyaniline via the formation of polyaniline-clay nanocomposite materials. Chemistry of Materials, 13, 1131-1136 (2001). DOI: $10.1021 / \mathrm{cm} 000938 \mathrm{r}$

[11] Fang J., Xu K., Zhu L., Zhou Z., Tang H.: A study on mechanism of corrosion protection of polyaniline coating and its failure. Corrosion Science, 49, 4232-4242 (2007).

DOI: $10.1016 /$ j.corsci.2007.05.017

[12] Hung W-I., Chang K-C., Chang Y-H., Yeh J-M.: Advanced anticorrosive coatings prepared from polymerclay nanocomposite materials. in 'Advances in nanocomposites - Synthesis, characterization and industrial applications' (ed.: Reddy B.) InTech, Rijeka, 561-582 (2011).

DOI: $10.5772 / 15355$

[13] Chang K-C., Hsu M-H., Lu H-I., Lai M-C., Liu P-J., Hsu C-H., Ji W-F., Chuang T-L., Wei Y., Yeh J-M., Liu W-R.: Room-temperature cured hydrophobic epoxy/ graphene composites as corrosion inhibitor for coldrolled steel. Carbon, 66, 144-153 (2014).

DOI: $10.1016 /$ j.carbon.2013.08.052

[14] Ou J., Hu W., Xue M., Wang F., Li W.: Superhydrophobic surfaces on light alloy substrates fabricated by a versatile process and their corrosion protection. ACS Applied Materials and Interfaces, 5, 3101-3107 (2013). DOI: $10.1021 / \mathrm{am} 4000134$

[15] Momen G., Farzaneh M.: Facile approach in the development of icephobic hierarchically textured coatings as corrosion barrier. Applied Surface Science, 299, 41-46 (2014).

DOI: $10.1016 /$ j.apsusc.2014.01.179

[16] Yao H-B., Tan Z-H., Fang H-Y., Yu S-H.: Artificial nacre-like bionanocomposite films from the self-assembly of chitosan-montmorillonite hybrid building blocks. Angewandte Chemie International Edition, 49, $10127-$ 10131 (2010). DOI: 10.1002/anie.201004748

[17] Cheng Q., Li M., Jiang L., Tang Z.: Bioinspired layered composites based on flattened double-walled carbon nanotubes. Advanced Materials, 24, 1838-1843 (2012). DOI: $10.1002 / \mathrm{adma} .201200179$

[18] Wang J., Lin L., Cheng Q., Jiang L.: A strong bio-inspired layered PNIPAM-clay nanocomposite hydrogel. Angewandte Chemie International Edition, 51, 46764680 (2012). DOI: $10.1002 /$ anie. 201200267
[19] Cheng Q., Wu M., Li M., Jiang L., Tang Z.: Ultratough artificial nacre based on conjugated cross-linked graphene oxide. Angewandte Chemie International Edition, 52, 3750-3755 (2013). DOI: $10.1002 /$ anie.201210166

[20] Cheng Q., Jiang L., Tang Z.: Bioinspired layered materials with superior mechanical performance. Accounts of Chemical Research, 47, 1256-1266 (2014).

DOI: $10.1021 / \mathrm{ar} 400279 \mathrm{t}$

[21] Cui W., Li M., Liu J., Wang B., Zhang C., Jiang L., Cheng Q.: A strong integrated strength and toughness artificial nacre based on dopamine cross-linked graphene oxide. ACS Nano, 8, 9511-9517 (2014).

DOI: $10.1021 / \mathrm{nn} 503755 \mathrm{c}$

[22] Wang J., Cheng Q., Lin L., Jiang L.: Synergistic toughening of bioinspired poly(vinyl alcohol)-clay-nanofibrillar cellulose artificial nacre. ACS Nano, 8, 27392745 (2014).

DOI: $10.1021 / \mathrm{nn} 406428 \mathrm{n}$

[23] Yao H-B., Ge J., Mao L-B., Yan Y-X., Yu S-H.: $25^{\text {th }}$ anniversary article: Artificial carbonate nanocrystals and layered structural nanocomposites inspired by nacre: Synthesis, fabrication and applications. Advanced Materials, 26, 163-188 (2014).

DOI: $10.1002 / \mathrm{adma} .201303470$

[24] Cheng Q., Duan J., Zhang Q., Jiang L.: Learning from nature: Constructing integrated graphene-based artificial nacre. ACS Nano, 9, 2231-2234 (2015).

DOI: $10.1021 /$ acsnano. 5 b01126

[25] Gong S., Cui W., Zhang Q., Cao A., Jiang L., Cheng Q.: Integrated ternary bioinspired nanocomposites via synergistic toughening of reduced graphene oxide and double-walled carbon nanotubes. ACS Nano, 9, 1156811573 (2015).

DOI: $10.1021 /$ acsnano.5b05252

[26] Zhang Y., Gong S., Zhang Q., Ming P., Wan S., Peng J., Jiang L., Cheng Q.: Graphene-based artificial nacre nanocomposites. Chemical Society Reviews, 45, 23782395 (2016).

DOI: $10.1039 / \mathrm{C} 5 \mathrm{CS} 00258 \mathrm{C}$

[27] Xu Q. F., Wang J. N.: A superhydrophobic coating on aluminium foil with an anti-corrosive property. New Journal of Chemistry, 33, 734-738 (2009). DOI: 10.1039/B817130K

[28] Wang P., Zhang D., Qiu R., Hou B.: Super-hydrophobic film prepared on zinc as corrosion barrier. Corrosion Science, 53, 2080-2086 (2011). DOI: $10.1016 /$ j.corsci.2011.02.025

[29] Yuan S., Pehkonen S. O., Liang B., Ting Y. P., Neoh K. G., Kang E. T.: Superhydrophobic fluoropolymer-modified copper surface via surface graft polymerisation for corrosion protection. Corrosion Science, 53, 27382747 (2011). DOI: $10.1016 /$ j.corsci.2011.05.008

[30] de Leon A. C. C., Pernites R. B., Advincula R. C.: Superhydrophobic colloidally textured polythiophene film as superior anticorrosion coating. ACS Applied Materials and Interfaces, 4, 3169-3176 (2012). DOI: $10.1021 / \mathrm{am} 300513 \mathrm{e}$ 
[31] Lo T-Y., Huang Y-C., Hsiao Y-N., Chao C-G., Whang W-T.: Preparation of superhydrophobic polyimide films modified with organosilicasol as effective anticorrosion coatings. Surface and Coatings Technology, 258, 310 319 (2014).

DOI: $10.1016 /$ j.surfcoat.2014.09.008

[32] Liu Y., Li S., Zhang J., Liu J., Han Z., Ren L.: Corrosion inhibition of biomimetic super-hydrophobic electrodeposition coatings on copper substrate. Corrosion Science, 94, 190-196 (2015).

DOI: $10.1016 /$ j.corsci.2015.02.009

[33] Zhang X-F., Chen R-J., Hu J-M.: Superhydrophobic surface constructed on electrodeposited silica films by two-step method for corrosion protection of mild steel. Corrosion Science, 104, 336-343 (2016).

DOI: 10.1016/j.corsci.2015.12.028

[34] Yilgor I., Bilgin S., Isik M., Yilgor E.: Facile preparation of superhydrophobic polymer surfaces. Polymer, 53, 1180-1188 (2012).

DOI: $10.1016 /$ j.polymer.2012.01.053

[35] Liu T., Chen S., Cheng S., Tian J., Chang X., Yin Y.: Corrosion behavior of super-hydrophobic surface on copper in seawater. Electrochimica Acta, 52, 8003-8007 (2007).

DOI: $10.1016 /$ j.electacta.2007.06.072

[36] Wang N., Xiong D., Deng Y., Shi Y., Wang K.: Mechanically robust superhydrophobic steel surface with antiicing, UV-durability, and corrosion resistance properties. ACS Applied Materials and Interfaces, 7, 6260 6272 (2015).

DOI: $10.1021 /$ acsami.5b00558

[37] Yang T-I., Peng C-W., Lin Y-L., Weng C-J., Edgington G., Mylonakis A., Huang T-C., Hsu C-H., Yeh J-M., Wei Y.: Synergistic effect of electroactivity and hydrophobicity on the anticorrosion property of room-temperature-cured epoxy coatings with multi-scale structures mimicking the surface of Xanthosoma sagittifolium leaf. Journal of Materials Chemistry, 22, 15845-15852 (2012).

DOI: $10.1039 / \mathrm{C} 2 \mathrm{JM} 32365 \mathrm{~F}$

[38] Chen H., Zhang X., Zhang P., Zhang Z.: Facile approach in fabricating superhydrophobic $\mathrm{SiO}_{2} /$ polymer nanocomposite coating. Applied Surface Science, 261, 628-632 (2012).

DOI: 10.1016/j.apsusc.2012.08.071

[39] Kartsonakis I. A., Balaskas A. C., Koumoulos E. P., Charitidis C. A., Kordas G.: Evaluation of corrosion resistance of magnesium alloy ZK10 coated with hybrid organic-inorganic film including containers. Corrosion Science, 65, 481-493 (2012).

DOI: $10.1016 /$ j.corsci.2012.08.052

[40] Zhou C., Lu X., Xin Z., Liu J., Zhang Y.: Polybenzoxazine/ $\mathrm{SiO}_{2}$ nanocomposite coatings for corrosion protection of mild steel. Corrosion Science, 80, 269-275 (2014).

DOI: $\underline{10.1016 / j . c o r s c i .2013 .11 .042 ~}$
[41] Qing Y., Yang C., Hu C., Zheng Y., Liu C.: A facile method to prepare superhydrophobic fluorinated polysiloxane/ZnO nanocomposite coatings with corrosion resistance. Applied Surface Science, 326, 48-54 (2015). DOI: $10.1016 /$ j.apsusc. 2014.11.100

[42] Jie H., Xu Q., Wei L., Min Y.: Etching and heating treatment combined approach for superhydrophobic surface on brass substrates and the consequent corrosion resistance. Corrosion Science, 102, 251-258 (2016).

DOI: $10.1016 /$ j.corsci.2015.10.013

[43] Zhang D., Qian H., Wang L., Li X.: Comparison of barrier properties for a superhydrophobic epoxy coating under different simulated corrosion environments. Corrosion Science, 103, 230-241 (2016).

DOI: $10.1016 /$ j.corsci.2015.11.023

[44] Sun M., Luo C., Xu L., Ji H., Ouyang Q., Yu D., Chen Y.: Artificial lotus leaf by nanocasting. Langmuir, 21, 8978-8981 (2005).

DOI: $10.1021 / 1 \mathrm{a} 050316 \mathrm{q}$

[45] Weng C-J., Huang J-Y., Huang K. Y., Jhuo Y. S., Tsai M. H., Yeh J. M.: Advanced anticorrosive coatings prepared from electroactive polyimide- $\mathrm{TiO}_{2}$ hybrid nanocomposite materials. Electrochimica Acta, 55, 84308438 (2010).

DOI: $10.1016 /$ j.electacta.2010.07.063

[46] Chang K. C., Chuang T. L., Ji W. F., Chang C. H., Peng Y. Y., Shih H., Hsu C. L., Yeh J. M., Tang W. C., Su Y. C.: UV-curable nanocasting technique to prepare bioinspired superhydrophobic organic-inorganic composite anticorrosion coatings. Express Polymer Letters, 9, 143153 (2015).

DOI: $10.3144 /$ expresspolymlett.2015.15

[47] Cheng Q., Li M., Zheng Y., Su B., Wang S., Jiang L.: Janus interface materials: Superhydrophobic air/solid interface and superoleophobic water/solid interface inspired by a lotus leaf. Soft Matter, 7, 5948-5951 (2011). DOI: $10.1039 / \mathrm{C} 1 \mathrm{SM} 05452 \mathrm{~J}$

[48] Chong A. S. M., Zhao X. S.: Functionalization of SBA15 with APTES and characterization of functionalized materials. The Journal of Physical Chemistry B, 107, 12650-12657 (2003).

DOI: $10.1021 / \mathrm{jp} 035877+$

[49] Wang X., Lin K. S. K., Chan J. C. C., Cheng S.: Direct synthesis and catalytic applications of ordered large pore aminopropyl-functionalized SBA-15 mesoporous materials. The Journal of Physical Chemistry B, 109, 1763-1769 (2005).

DOI: $10.1021 / \mathrm{jp} 045798 \mathrm{~d}$

[50] Gadelmawla E. S., Koura M. M., Maksoud T. M. A., Elewa I. M., Soliman H. H.: Roughness parameters. Journal of Materials Processing Technology, 123, 133145 (2002).

DOI: 10.1016/S0924-0136(02)00060-2

[51] Park S-M., Yoo J-S.: Peer reviewed: Electrochemical impedance spectroscopy for better electrochemical measurements. Analytical Chemistry, 75, 455A-461A (2003).

DOI: $10.1021 / \mathrm{ac} 0313973$ 